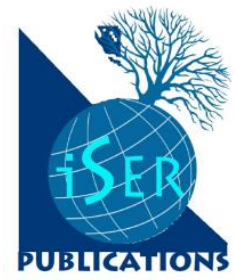

\title{
Mental Constructions for The Group Isomorphism Theorem
}

\author{
Arturo Mena-Lorca \& Astrid Morales Marcela Parraguez \\ Pontificia Universidad Católica de Valparaíso, CHILE
}

•Received 11 November $2015 \bullet$ Revised 12 December $2015 \bullet$ Accepted 01 February 2016

The group isomorphism theorem is an important subject in any abstract algebra undergraduate course; nevertheless, research shows that it is seldom understood by students. We use APOS theory and propose a genetic decomposition that separates it into two statements: the first one for sets and the second with added structure. We administered a questionnaire to students from top Chilean universities and selected some of these students for interviews to gather information about the viability of our genetic decomposition. The students interviewed were divided in two groups based on their familiarity with equivalence relations and partitions. Students who were able to draw on their intuition of partitions were able to reconstruct the group theorem from the set theorem, while those who stayed on the purely algebraic side could not. Since our approach to learning this theorem was successful, it may be worthwhile to gather data while teaching it the way we propose here in order to check how much the learning of the group isomorphism theorem is improved. This approach could be expanded to other group homomorphism theorems provided further analysis is conducted: going from the general (e.g., sets) to the particular (e.g., groups) might not always the best strategy, but in some cases we may just be turning to more familiar settings.

Keywords: APOS theory, equivalence relation, genetic decomposition, group isomorphism theorem

\section{INTRODUCTION}

Let $G_{y} G^{d}$ be groups, and let $f: G \rightarrow G^{\prime}$ be a group homomorphism. The (sometimes called "first") group isomorphism theorem (GIT) states that $\operatorname{Im}(f)=f(G)$ is isomorphic to the quotient group $G / \operatorname{Ker}(f)$ of $G$ over the kernel of $f$. The GIT is the first of a dozen or so homomorphism theorems that are used to build groups from others already known, to identify groups and determine their structure, to examine the solution of equations by radicals, and so forth. These theorems have immediate extensions to other theories whose mathematical objects have underlying groups-rings, vector spaces, topological groups, and so on. Thus, this is a very important subject for mathematics majors.

Mathematical requirements for the theorem are many. Also, from a theoretical perspective that will be made explicit later on, one should add specific information on the mental constructions related to each one of those requisites. This refines the initial view on the complexity of the GIT, but also provides some hope about the attainability of its learning: in fact, some research has shown that it is not always

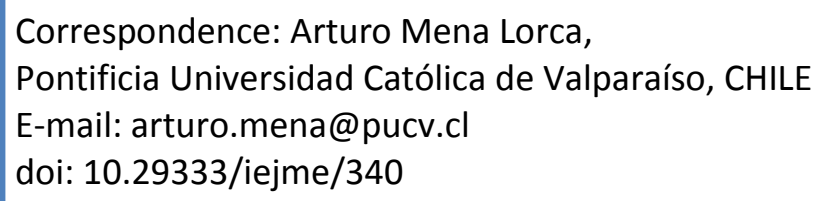


imperative to totally apprehend a mathematical concept in order to understand another that is built on it (Brown, De Vries, Dubinsky, \& Thomas, 1997).

\section{LEARNING OF THE GIT AND RELATED SUBJECTS}

Literature on teaching and learning of abstract algebra is scarce as compared to other areas of mathematics teaching and learning (see, for example, Thomas, de Freitas, Huillet, Ju, Nardi, Rasmussen \& Xie, 2015). Only a few studies concern our subject, and many are the work of RUMEC-related researchers. ${ }^{1}$ (Weber, 2002; Iannone \& Nardi, 2002; Novotná, Stehlíková \& Hoch, 2006; Ioannou \& Nardi, 2009; Weber \& Larsen, 2008; and Larsen, 2009, 2013a) dealt with elementary aspects of group theory, such as binary operation, subgroup, the notions of homomorphism and isomorphism; (Larsen, 2013b) added normality and quotient groups; (Ioannou, \& Iannone, 2011) included aids to visualization of cosets and of the TIG. The more recent works address how group theory concepts are developed from students' informal knowledge, and include also affective issues. Closer to our approach, (Asiala, Dubinsky, Mathews, Morics, \& Oktaç, 1997; and Lajoie, 2001) are the only works that we know of that have focused on normality, cosets and quotient groups and given corresponding APOS' genetic decompositions - to be described later on.

There is consensus among researchers in that, in general, the teaching of abstract algebra cannot be considered a successful endeavor (Leron \& Dubinsky, 1995; Lajoie, 2001; Godfrey \& Thomas, 2008; Stadler, 2011); its difficulty seems to come from the abstract nature of the objects involved (Hazzan \& Leron, 1996). Furthermore, it may drive off from the subject even students who had thus far been well motivated (Clark et al., 1997). Dubinsky, Dauterman, Leron, and Zazkis (1994) have shown that the main problem in this area is that while students must work with abstract concepts and write proofs, they lean heavily on canonical procedures. This questions the residual knowledge of the teaching of the subject and shows that students need more help in learning how to build certain concepts and results and understanding what they have built (Asiala et al., 1996).

The GIT's importance notwithstanding, it is common lore that it is a "difficult" subject: many undergraduate students would agree and many instructors complain about the low level of results from teaching it. That lore is confirmed by research; for instance, Brown et al. (1997) showed that most students do not grasp what a quotient $G / N$ is nor that its structure depends on the normal subgroup $N$ in $G$, and that they are unable to define functions from $G / N$. As shown also by Nardi (1996), the theorem thus remains unreachable to the learner.

The notions of normal subgroup and quotient group still represent a major problem for a newcomer, traceable to their introduction by Galois, circa 1830 (Galois, 1897). In 1869, Camille Jordan started a commentary on Galois' work that he later extended in his book (Jordan, 1869, 1872). That year, Sophus Lie met Felix Klein in Berlin, and they started a joint work that continued in Paris in 1870. They were deeply impressed by the work of both Galois and Jordan (Bourbaki, 1999); as it is well known, Klein used it in the Erlangen Programme and Lie in the creation of structures nowadays called Lie Groups and Lie Algebras. Nevertheless, Bourbaki (2006) adds: "Truth to tell, Klein and Lie must both have had difficulty in penetrating this new mathematical universe," and quotes Klein saying that Jordan's work seemed to him a book "sealed with seven seals" (p. 287).

Galois' change of the approach to solving equations is far-reaching: he did not aim directly to find the solutions, but looked for the group structure $G$ that they form: if $G$ has a normal subgroup $H$, the equation can be solved by means of $H$ and a group $G / H$ that results from a partition of $G$ induced by $H$ (for him, the group of an auxiliary equation). 
Burnside (1897) then proved the GIT. This theorem may also serve as a sample of the process started by Galois: the study of equations reached a trans-operational level in the sense of Piaget and García (1989), which meant that, from the study of equations, algebra evolved into the study of (algebraic) structures (Mena-Lorca, 2010). It cannot be claimed that students understand this change of perspective. (See, for example, Novotná, Stehlíková, \& Hoch, 2006; Stadler, 2011).

Now, it can be argued that normal subgroups and quotient groups are epistemological obstacles in the sense of Brousseau (1998); in particular, the difficulties that students encounter when studying them are intrinsic to those subject matters (Mena-Lorca, 2010); and, albeit the concepts nowadays used are more clear and argumentation is somehow simpler, those subjects remain remote to the students. Of course, addressing this is not just a matter of going back to equations, since abstract algebra relates also to other subjects - some of which we have already mentioned - and, more importantly, because this would tend to increase the difficulty, since it involves that complex transition of the conception of algebra itself as a discipline

Accordingly, one should think about a way to relate better this mathematics to the students' intuition.

Now, the very nature of the TIG is twofold: there is a bijection (a set-theory fact), and this bijection is an isomorphism (an algebraic fact). Similarly, a quotient $G / H$ is a set, which, $H$ being normal in $G$, inherits a group structure.

Moreover, it turns out that, if one 'forgets' the algebraic structure, the quotientpartition idea is very simple and customary-not only in the realm of mathematics-and similarly for a set version of the GIT (see Mena-Lorca, 2010). Also, provided that the students acquaint themselves with these, and since a priori there is no reason to expect that a quotient is a group, the need for normality in the group case might be better understood.

That notwithstanding, the group structure of the quotient may remain hard to get, but, to approach it, the abelian case-the simplest one-is useless; still, as we

will see, the quotient group $\mathbf{G} / \operatorname{Ker}(\mathbf{f})$ is closer to the students' intuition than the general case. After examining some cognitive aspects that had to be consideredand that we will describe later on-this is the way we have chosen.

\section{APPROACHING THE GIT}

For the sake of precision as well as clarity, from this point on, unless stated otherwise, $(G, e),\left(G^{\prime}, e^{\prime}\right)$ are groups, $|G|=n ; a, b \in G ; f: G \rightarrow G^{\prime}$ is a group homomorphism of kernel $\operatorname{Ker}(f)$ and image $\operatorname{Im}(f) ; H \unlhd G ; a H=\{a h: h \in H\}=\bar{a} ; G / H=\{a H: a \in G\} ; \bar{f}: G / H \rightarrow G ; \bar{f}(\bar{a})=f(a)$

Remark: All mathematical concepts and results of this section but the set isomorphism theorem can be found in (Hungerford, 2003).

Our experience shows that many students learning the GIT proceed according to their understanding of what "algebra" is and do not address certain key facts. For example, in dealing with $G / H$, they undertake the proof of group properties of the operation $\bar{a} \bar{b}=\overline{a b}$, but most show no concern about the necessity of independence of the operation from the representatives taken in the cosets.

We analyzed a number of textbooks currently in use that deal with group theory (Fraleigh, 2003; Herstein, 1999, Hungerford, 2003, Lang, 2005): when treating quotient groups, each starts from the construction of the cosets $a H_{,} a \in G$, gathers them together in $G / H$, and then proceeds to the operation $a H b H=a b H$. (See, for example, Fraleigh. 2003, Dummit \& Foote, 2011). We also interviewed researchers 
in abstract algebra that lecture on the subject at the top universities in the country and found that they proceed in basically the same way. ${ }^{2}$ Thus, the construction of quotients is treated as a purely algebraic matter.

On the other hand, it is well known that mathematics (and sciences in general) permanently and variously uses partitions and equivalence relations (ERs). The same is true for every individual who uses them extensively in every aspect of his work and daily life: classifying and considering different objects as equivalent occurs throughout every day (Mena-Lorca, 2010).

Nevertheless, it seems that when learning about the GIT, students work with quotients for the first time in a formal way: cosets are presented as a purely algebraic and abstract notion, and the student has to construct them and then reunite all of them in another purely abstract object-the "quotient group"—all of them remote from more familiar objects that they could draw on. Moreover (often even before comprehending the quotient as a set), they must consider an algebraic structure on it and define a homomorphism from it. Faced with this situation, they rush to check properties as we described earlier and do not exhibit an understanding of the nature of the partition they are dealing with (Mena-Lorca, 2010).

Thus, based on this preliminary evidence, from a cognitive perspective we expected that, when dealing with our subject, it would be more natural to first build quotients (i.e., partitions) and then examine the possibility of inducing a structure on $G / H$ compatible with the operation on $G$.

For such an approach, we need to consider a theorem that we will call ER/P that states that every ER defined on a set $S$ induces a partition on $S$ and that, conversely, every partition on $S$ defines an ER on $S$. In this regard, it is interesting to note that students, even those who lack a precise notion of ER and/or of partition, spontaneously use an informal version of ER/P (as a theorem in action; see Vergnaud, 1981), operational to the point that often they do not distinguish between partitions and equivalence relations defined on a certain set (Mena-Lorca, 2010).

In the algebraic setting, any subgroup $H$ of $G$ defines two ERs on $G$, namely $a R_{H_{n} l} b: a^{-1} b \in H$ (left) that induces the partition $G / H=\{a H: a \in G\}$, and $a R_{H, r} b: a b^{-1} \in H$ (right), which in turn induces the partition $G \backslash H=\{\mathrm{Ha}: a \in G\}$. If $H$ is normal in $G$, both ERs coincide, and both partitions coincide- $a H$ being always equal to $H a$. Now, $H=\operatorname{Ker}(f)$ is, in fact, a normal subgroup of $G$; the corresponding ER is $a R_{\operatorname{Ker}(f)} b: a^{-1} b \in \operatorname{Ker}(f)$, and we have $a R_{\operatorname{Ker}(f)} b \Leftrightarrow a^{-1} b \in \operatorname{Ker}(f) \Leftrightarrow f\left(a^{-1} b\right)=e^{\sigma} \Leftrightarrow f\left(a^{-1}\right) f(b)=e^{*} \Leftrightarrow f(a)^{-1} f(b)=e^{*} \Leftrightarrow$ $f(b)=f(a)$

Thus, $R_{\operatorname{Ker}(f)}: R_{f}$ is defined by $a R_{f} b: f(a)=f(b)$, and $G / \operatorname{Ker}(f)$ simply gathers together in an equivalence class the elements that have the same image under $f$; thus, $\bar{a}=\left\{x \in G: x R_{f} a\right\}=a \operatorname{Ker}(f)=\operatorname{Ker}(f) a$. Now, let $G, G^{\prime}$ be any sets and $f: G \rightarrow G^{\prime}$ be any function. Then $f$ defines an ER on $G$ by $a R_{f} b: f(a)=f(b)$ and it is immediate that $\left\{x \in G: x R_{f} a\right\}=\bar{a} \rightarrow f(a)$ defines a bijection from $G / R_{f}$ to $\operatorname{Im}(f)$. This last statement we call the set isomorphism theorem (SIT). ${ }^{3}$ We can use the SIT as a lemma for GIT. Also, a student may realize that the SIT is abundantly encountered-for instance, prices in a store are not set for individual objects but for equivalence classes of objects and so forth.

Our working hypothesis is that the learning of the GIT is simplified and achieved if the set theoretical and the properly algebraic parts are separated. By this we do not only mean to consider first the partitions $G / H$ (whose cosets are characterized by having the same cardinal number) but, as in the statement of the SIT, any partition 
on the set $G$. Thus, students have the opportunity to draw upon their naive, nonformal knowledge of ERs and partitions and only then, in an already more familiar environment, worry about the correct definition of operations and functions.

Our study, then, apart from verifying if the approach proposed is suitable, should also regard: if the students freely use ERs, partitions and the theorem ER/P that relates them (due to preliminary evidence, we are confident about this); whether their approach to the GIT can draw on that; and additionally, if, in case they approach the (re)construction of the theorem in purely algebraic terms, whether they can succeed in it.

\section{THEORETICAL FRAME}

We used the APOS theory that was devised by Dubinsky in the 80s (Dubinsky 1986; Arnon et al., 2014). He bases this theory on Piaget's reflective abstraction to describe the construction of mental objects, and he distinguishes different types of it, or mechanisms: internalization, coordination, encapsulation, generalization, and reversal. These are the origin of different (mental) constructions: Actions, Processes, Objects, and Schemas - whence the APOS acronym.

Let us consider a fragment $\boldsymbol{F}$ of mathematical knowledge in Dubinsky's perspective (as in Arnon et al., 2014): Individuals have an Action conception of $\boldsymbol{F}$ if the changes that they make on it are done step by step, obeying stimuli that are and are perceived as external. Individuals interiorize an Action in a Process concept of $\boldsymbol{F}$ if they can perform an internal operation that does (or that they imagine does) essentially the same transformations entirely in their mind, not necessarily covering all the specific steps. They can coordinate two or more Processes, or reverse one to obtain a new Process. If individuals think about the Process as a whole and build and perform transformations on the whole Process, they have encapsulated it in an Object conception of $\boldsymbol{F}$. If they need to return from the Object conception to the Process from which it comes from, they do it by de-encapsulating the Object. A Schema of $\boldsymbol{F}$ is a collection of Actions, Processes, Objects, and other Schemas that are consciously or unconsciously related in the mind of the individual in a coherent cognitive structure. Coherence relates to recognizing relationships within the Schema, recognizing whether the Schema can solve a particular mathematical situation, and using it in such a case. In dealing with a mathematical problem, the individual recalls a Schema and unfolds it to gain access to its constituents, uses relationships between them, and works with the whole. A Schema is ever evolving and can be considered as a new Object to which an individual can apply Actions and Processes; in such a case it is said that the Schema has been thematized.

APOS methodology has been consolidated since the 80s and it is considered a successful method of research in the area of abstract algebra learning today (Dubinsky \& Lewin, 1986; Dubinsky, 1991; Asiala et al., 1996; Brown et al., 1997; Trigueros \& Oktaç, 2005, Arnon et al, 2014). It uses a Research cycle that aims to allow for empirical evidence of the mental mechanisms and constructions to be put into play in the construction of a fragment of mathematics: theoretical analysis, design, and implementation of instruments and data analysis and verification (Asiala et al., 1996, Arnon et al. 2014).

A genetic decomposition is a hypothetical model whose aim is to describe both the mental structures and mechanisms that a student might need-a genetic decomposition is not necessarily unique-in order to learn a specific fragment of mathematics (Arnon et al. 2014), and how those mechanisms and structures are organized in a Schema. It includes prerequisite structures that the students need to have constructed previously - that is, not just a list of concepts and facts that should be 'known' by them, but whether they are required as Actions, Processes, Objects or 
Schemas. Specific aspects, such as coordination of Processes, shed light on the constructions made by the students. Thus, a genetic decomposition not only deals with the cognitive aspects of the apprehension of knowledge by the students, but also clarifies the role of its prerequisites - in fact, it is common knowledge that, when students approach specific subjects, their mathematical prerequisites are not necessarily well known by them. (Arnon et al. 2014). It typically starts as a preliminary genetic decomposition, which has to be validated, often after adjustment based on empirical data (Asiala et al., 1996). A preliminary genetic decomposition is based on "the researchers' experiences in the learning and teaching of the concept, their knowledge of APOS theory, their mathematical knowledge, previously published research on the concept, and the historical development of the concept" (Arnon et al., 2014, p. 28).

In what follows, we report some relevant findings on mental constructions related to groups, normal subgroups, and quotient subgroups obtained by using APOS theory.

We assume that the student has encapsulated the general concepts of group (Brown et al., 1997) and of function, epijectivity, and injectivity (Dubinsky, 1991; Baker, Trigueros, \& Hemenway, 2001). Hamdan (2006) presents an initial genetic decomposition for ERs (and functions). An explicit genetic decomposition for (an additive group) homomorphism can be found in Roa-Fuentes and Oktaç (2010). Isomorphism is studied in Leron, Hazzan, and Zazkis (1994, 1995); Leron and Dubinsky (1995); Dubinsky and Zazkis (1996); and Nardi, (1996, 2000). Construction and multiplication of cosets is seen in Dubinsky et al. (1994). Asiala et al. (1997) report at length about the mental constructions on cosets and normality: roughly two thirds of the students considered were successful in constructing cosets and normality, while for quotient groups the rate dropped to around one third and, generally speaking, a high percentage of the students seems lost and can end up disconnecting from the subject (see also Ioannou \& Nardi 2009). In fact, some researchers doubt the permanence in time of the quotient concept (Dubinsky et al., 1994). Note that if the encapsulation of the quotient-partition is not achieved it is certainly doubtful that the concept has some value for the student. Dubinsky (1986) showed that students' difficulties with mathematical symbolism (e.g., multiplying cosets $a H$ ) come from trying to apply labels before achieving encapsulation.

We found no genetic decomposition for the GIT.

\section{CONSTRUCTING THE GIT}

We turn now to requirements for achieving an Object conception of the GIT. According to the research cited above, one needs Object conceptions of set (for group), multiple quantification (for isomorphism), function (for homomorphism), group (as a homomorphism is an Action on a group), subgroup (examining normality requires its encapsulation), and homomorphism (examining bijectivity requires its encapsulation). We also need Object conceptions of $\operatorname{Ker}(f)$ (to get $G / \operatorname{Ker}(f)$ ) and $\operatorname{Im}(f)$ (to compare it with $G / \operatorname{Ker}(f)$ ). A Schema conception of group consisting of Object conceptions of set, group, subgroup and function as well as a Process conception of binary operation (the coordination of set and binary operation plays a fundamental role in the Schema) are also necessary. A genetic decomposition for the GIT does not need the general concept of quotient group, but of $G / \operatorname{Ker}(f)$, and working with this particular case might help the construction of the general case. Also, the encapsulation of cosets just as sets does not need to be carried out by an "algebraic" process: the encapsulation of a quotient set is first achieved as the partition $G / \operatorname{Ker}(f)$ defined by $a R_{f} b: f(a)=f(b)$ and then 
defined as an operation on $G / \operatorname{Ker}(f)$. As shown in the research cited above, this is not an easy task, but in this approach students are able keep in mind that they are dealing with (sub)sets of elements to start with. Moreover, we did not require an Object conception of $G / \operatorname{Ker}(f)$ but a Schema composed of Object conceptions of group, subgroup (and set, function, and binary operation), and (the naive version of) $\mathrm{ER} / \mathrm{P}$.

Over those constructions, the student is supposed to make a number of coordinations-several of them already established in the literature reviewedsuch as (in this case, $N=\operatorname{Ker}(f), G / N=G / \operatorname{Ker}(f)$ )): subgroup with cosets (as classes in a partition) through the normality property in order to obtain a Process conception of a normal subgroup; group with $\mathrm{E} / \mathrm{R}$, and subgroup and quotient group in order to obtain a Process conception of $G / N$; function and binary operation, so as to construct the homomorphism as a Process which encapsulates by means of the identification of the quotient group and its image; function with ER/P in order to define a function from the quotient, then a bijection and obtaining the SIT; etc.

\section{METHOD}

We designed and implemented some instruments to explicitly reflect the constructions through which students can grasp the concepts that are needed. For this, we framed our preliminary genetic decomposition using a case study methodology (Goetz \& Le Compte, 1988; see also Arnon et al., 2014) so as to provide, through a purposeful selection of participants, knowledge about the learning of the GIT through the study of mental mechanisms and constructions shown by comparatively advantaged individuals in dealing with our subjects.

We started with four groups of students, 17 in total, each group from a different university in Chile. The universities were chosen so as to ensure: equivalence in admissions processes in terms of basic skills for addressing the mathematical field, existence of both an undergraduate program in mathematics teaching that included group theory, existence of a graduate program in mathematics, and affordability for the study. The students were required to have approved a course in abstract algebra.

We designed a questionnaire (see Appendix) to be administered to the aforementioned students. The answers to the questionnaire were not aimed at determining which students might proceed with the construction of the GIT, but rather which might not. It was applied in a classroom at the location where the students' programs are offered and lasted about one hour. At least one of the researchers was in the room, and provided explanations about symbols or related questions (e.g., the meaning of "inside" and "outside" mathematics in Question 4 of the questionnaire) but not prompting.

Then we applied two semi-structured interviews to students chosen based on their answers to the questionnaire. This type of interview is customarily used in order to be able to interact with the interviewee and to be able to clarify answers if needed (Dubinsky et al., 1994). For the seven students selected in Group 1 (Juan, José, Pedro, Luis, Jorge, Ana, and Manuel), who we could not assume had paid attention to equivalence relations and partitions, we used Script 1 (see Appendix). For the three students in Group 2 (Carlos, Marta, and Leslie) we used Script 2 (see Appendix), since we knew that they had treated equivalence relations and partitions in their curricula. The aims of these interviews were to gather information about the viability of our preliminary genetic decomposition and to refine it if necessary, as well as determining the difficulties encountered by students in dealing with our theorem.

The interviews were individual, were held in isolated rooms at the location of the academic units to which the students belonged, and were videotaped. Each 
interview lasted an hour and a half and in all but one of them there were at least two researchers present. The whole team reviewed the videotapes.

\section{Questionnaire}

Given the limited aim of the questionnaire, it was not tested beforehand: students' inability to proceed with the construction of the GIT would be shown in the corresponding interviews. Thus, we do not claim that students who did not do well in the questionnaire would not be able to end up constructing the GIT, but that we doubt it, and that it was preferable to interview others.

There were two kinds of questions (enumeration refers to the questionnaire):

A. The students should:

- distinguish between elements of a group and sets of them (1a, b, c): if they do not, they would not be able to consider cosets as elements not of $G$ but of a quotient of $G$, which is crucial for defining the isomorphism of the GIT;

- realize that the operation of a subgroup is the restriction of the operation on $G$ (1d): otherwise, since it is needed that the operation on cosets of $G$ has to be induced by the operation on $G$, the students would lose track of the structure they have to deal with;

- have an idea, though vague, of normality (2a): if not, it is unlikely that they would be able to approach the need for the operation in the classes to be well defined;

- be acquainted with the importance of ERs and partitions inside and outside mathematics, or at least be able to come up with some examples (4): this is needed to take advantage of the set isomorphism theorem for the construction of the GIT;

- be able to at least generally express the notions of ER and partition or produce an approach to them, even in an informal way (5a, b): also to make use of the set isomorphism theorem;

- realize or consider that there is a connection between ERs and partitions defined over the same set (5c): the GIT needs for a translation of the ER defined by $\operatorname{Ker}(f)$ on $G$ into cosets defining $G / \operatorname{Ker}(f)$;

- be able to define homomorphisms in "simple" cases (6): even though this does not guarantee to be able to define an isomorphism starting from a quotient, its absence suggests that the students will not succeed in this last one;

- realize that the commutative property is "preserved" by isomorphism (7): thinking that a non commutative group may be isomorphic to a commutative one suggests that the acting idea of isomorphism is only related to bijection and not to the group structure.

B. We would welcome (but not require) that the student:

- have an idea about why operations on cosets work (2b);

- have a possibility of analyzing normality for subgroups of a small group and (even) of the construction of a quotient group (2b);

- show an idea about how to identify isomorphic groups that are not stated in the same way (8).

At this stage, we valued any arguments, even if not formally stated. Based on experience and research, our expectations for the answers were rather low, and we did not ask for the GIT.

We collected the questionnaires and tabulated them in a colored matrix in order to be able to easily glance at the correctness of the answers of each student to each question. We then decided which individuals to interview by means of identifying correct answers in most questions other than $2 b, 3$, and 8; "good" answers on these 
three questions would prevail over some wrong answers in the others. We did not proceed using a checklist, nor did we hypothesize that the selected students had clarity on the requisites stated above as that would be determined in the interviews.

\section{Interviews}

We did not wait for interviewees to fluidly answer our questions, which would have been contrary to the existing research as well as our own experience. As stated earlier, the scripts focused on examining the eventual presence of mental constructions and mechanisms that would come into play in connection with the GIT as we propose it: on the one hand, some aspects of group theory, which we would require even in a rather vague form, and, on the other, questions related to ER/P.

The scripts were used as a broad guide for the researchers' APOS semi-structured interviews. Notations were explained as needed. Interviewees were asked to explain their thoughts, to draw, and to give examples. The tone was conversational.

Script 1 began by asking about the GIT and we did not expect a clear answer on it -in fact, our experience suggested that most students would have forgotten about it, a result that was shown in the interviews. We then asked interviewees to elaborate on ERs and partitions. The key issues in our approach were that we expected subjects to realize were that, given $f: A \rightarrow C$ a function, $f$ defines an ER $R_{f}$ on $A$, and that there is a one-to-one correspondence between the quotient $A / R_{f}$ and $f(A)$. We asked this, but with low expectations. Thus, if students in Group 1 were not able to do this, we gave them the definition of $R_{f}$ in order to see whether they could elaborate from there, that is, relate it to the quotient defined by $\operatorname{Ker}(f)$ on $G$ and thus proceed to the GIT.

Script 2 was prepared for interviews that we expected to proceed in a more direct course.

\section{ANALYSIS AND DISPLAY OF SOME RESULTS}

As expected, every interviewee showed Process conceptions of group, subgroup, and normal subgroup. Luis, Ana, Manuel, and Marta manifested Object conceptions of group, subgroup, and normal subgroup and were able to construct a quotient group. Pedro, Juan, and José could not progress much in their interviews and they could not elaborate on examples nor on drawings: Juan and José tried instead to recollect definitions (such as Kernel, image, partition) and theorems (such as " $\left.(G / H) /(K / H)=G / K^{\prime \prime}\right)$. On the other hand, everyone used ER/P as a theorem in action: when arguing, they changed from ERs to partitions and vice versa. Group 1 students could not elaborate much on the GIT, as expected; Ana was an exception. Students in Group 2 tended to be more assertive in stating the GIT and reasoning about it. We will turn to these later.

\section{Coordination}

We were able to verify the coordination that we expected. We give some examples and brief comments:

Subgroup and quotient group. José drew the usual picture of a set, labeled it $G / \sim$, and wrote " $x, y \in G: x \sim y \Leftrightarrow x y^{-1} \in H^{w}$. On the contrary, Jorge did not: he stated "A normal subgroup $N$ of a group $G$ is a group with certain properties." As we expected, Jorge could not elaborate on quotients.

Normal subgroup and quotient group. Ana correctly wrote $N \unlhd G: g n g^{-1} \in H, g \in G, h \in G$; and added: "They serve to construct the 
quotient groups. It is known that if $G$ is an abelian group and $N$ is a subgroup of $G$, then $N$ is a normal subgroup." (We will turn to her later).

Function and homomorphism. The absence of this coordination seems to impede the construction of the GIT. For instance, Pedro wrote " $\varphi:\left(G_{1}, \star\right) \rightarrow\left(G_{2}, \square\right)$; $g \rightarrow \varphi(g)^{n}$. We then had the following exchange:

Pedro: But ... the interesting [point] is that I need the function to be bijective in order to obtain an isomorphism.

Interviewer: You told me that you cannot prescind from the function concept.

Pedro: Oh, but I can prescind from the group concept.

Interviewer: Why?

Pedro: Why can I prescind from the group concept? Because the isomorphism concept is more general, uh...

On the contrary, Luis wrote, not quite correctly: $f: \mathbf{Z} \rightarrow \mathbf{Z} ; z \rightarrow 2 z ; f(a+b)=2(a+b)=2 a+2 b=f(a)+f(b) ; \operatorname{Ker}(f)=$ $\{0\} ; g \operatorname{Ker} f g^{-1}=\operatorname{Ker} f \Rightarrow \operatorname{Ker} f$ Normal; $^{\prime} \mathbf{Z} / \operatorname{Ker}(f) \simeq \mathbf{Z}$.

Nevertheless, his explanation showed just a Process GIT. Thus, the coordinating function and the homomorphism property would not suffice for an Object GIT-as one might expect.

Normal subgroup, quotient group, and homomorphism. We think that coordinating these Processes allows getting close to Object GIT. Manuel correctly stated the GIT, wrote it, and explained: $f: Z \rightarrow\{-1,1\}, f(x)=1, x$ even, $f(x)=-1, x$ odd, and then wrote $\operatorname{Ker}(f)=\{x \in \mathbf{Z}: f(x)=1\}=2 \mathbf{Z} ;\{-1,1\} \simeq \mathbf{Z} / 2 \mathbf{Z} \simeq \mathbf{Z}_{2}$. Nevertheless, he could not elaborate on ERs and partitions and he could state neither the SIT nor the GIT.

\section{Suitability of the Genetic Decomposition}

Ana's work. Ana, in Group 1, showed clarity in several aspects of the GIT. She was interviewed again with Script 2 in order to determine whether she could complete her approach but she could not. She correctly stated the SIT, but when asked about an eventual relationship with the GIT, she added, "The guarantee that one has in sets is that in defining the equivalence relation, partitions can be known and one can easily see the quotient, while for groups we do not have the equivalence relation." Thus, she could not coordinate the SIT with the structure of the group.

Carlos's work. Carlos, when asked for examples of ERs, both in mathematics and in real life, easily provided several ERs and partitions. Questioned on it, he stated $\mathrm{ER} / \mathrm{P}$ and explained how to define an ER from any partition. He was then given a diagram where two sets, $A, B,|A|=8 ;|B|=6$, are shown and was asked to draw a general function on it. He filled in the picture as shown in Figure 1.

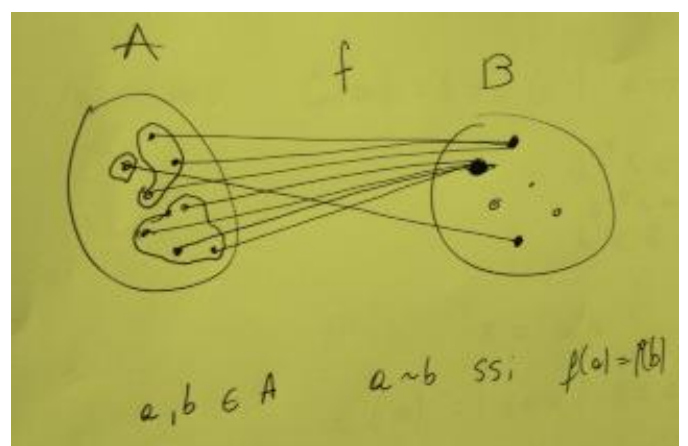

Figure 1. Carlos's work ("ssi" stands for "iff"). 
Asked whether he saw an ER in the picture, Carlos wrote below it as shown in Figure 1, which he correctly explained. When we inquired whether he saw another function in the picture, he wrote " $g: P \rightarrow B, g\left(P_{i}\right)=b_{i}{ }^{\prime}, P$ being the partition induced by $g$. He explained this correctly, although he called $P_{i}$ "a partition," as often happens. In answering what kind of function is $g$, he said that it is injective and that it is epijective over its image.

Turning to groups, we had to remind him what $H \unlhd G$ means and we gave him the definition of $R_{H}$. He checked the ERs properties. When asked, he wrote the definition of $\operatorname{Ker}(f)$. His response to our subsequent inquiry about the corresponding ER is shown in Figure 2.

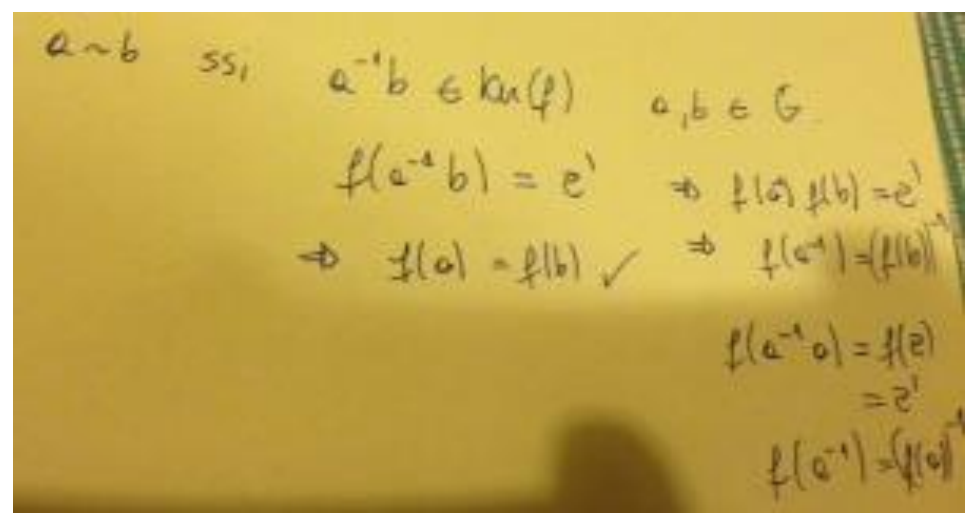

Figure 2. Carlos's work. (In the third column, he checked what he claimed in the second).

We then asked for $G / \operatorname{Ker}(f)$ and he gave this response, "Two elements are related when they have the same image, which was what we did at the beginning." He explained that the SIT and the GIT are quite similar, the difference being the need for group homomorphisms.

Thus, although Carlos could not work out the GIT by himself, it is clear that if he was given some definitions, he had a clear view of what had to be done. Moreover, he was able to argue using one figure where both the group $G$ and the quotient $G / H$ coincided, which is one of the problems that students have to overcome when reasoning about the GIT.

Carmen's approach. Carmen completely showed that our preliminary genetic decomposition works: her coordinations were readily apparent. Asked for an RE in either mathematics or in common life she immediately said, "What catches my attention more is the one of the pre-images." She wrote " $f: A \rightarrow B, f^{-1}(b)^{\mathrm{w}}$, and stated that an ER is defined by inverse images of elements of $B$. Asked for more examples, she gave one about buses with a common route and added, "If we think as a partition it comes out much more easily." She stated and explained ER/P. We then gave her a diagram, $A, B,|A|=11_{;}|B|=8$ and asked for a general function, and her response is as shown in Figure 3. 


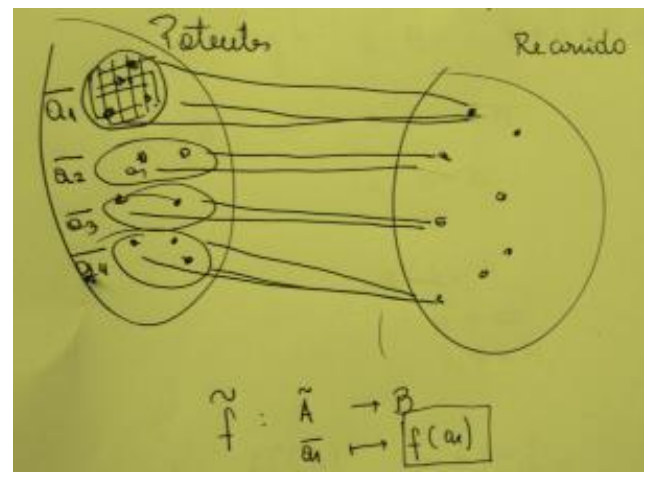

Figure 3. Carmen's work. (Patentes: license plates; Recorrido: route)

When asked for another function that she may perceive in that setting, she explained that, for example, bus fares are not fixed for individuals but for sets: buses on the same route have the same fare. We then asked her to generalize what is occurring, that is, a sort of theorem that can be stated. Carmen then correctly stated our SIT. When we talked about a homomorphism between two groups, she wrote " $f: G_{1} \rightarrow G_{2}$ ". She remembered that the definition of $H \leqq G_{1}$ was "Ker $(f)=f^{-1}\left(1_{2}\right)^{"}$, and stated that the equivalent classes in $G / \operatorname{Ker}(f)$ are " $b \operatorname{Ker}(f), b \in G_{1}{ }^{"}$. She also wrote ${ }^{\bar{"}} f: G / \operatorname{Ker}(f) \rightarrow \operatorname{Im}(f) ; a \operatorname{Ker}(f) \mapsto f(a)^{u}$, and stated that it is an isomorphism. We then had the following exchange:

Interviewer: This theorem is called the GIT. What would you call to the one that you made at the beginning?

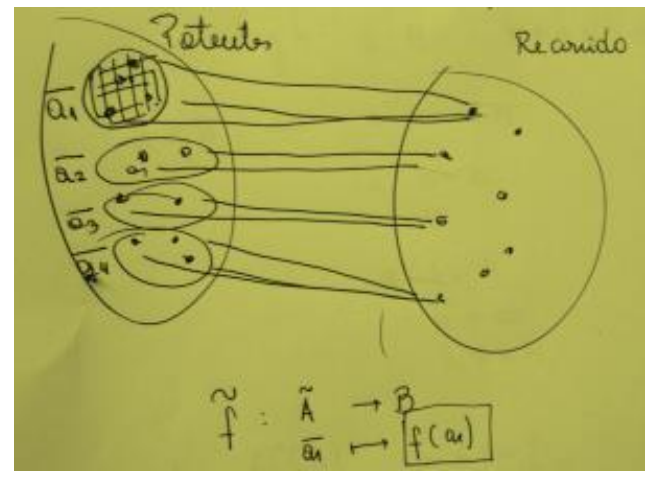

Figure 3. Carmen's work. (Patentes: license plates; Recorrido: route)

Carmen: Phew!... of the isomorphism because it has to have a form... Here there is no form since there is no structure... but what could it be; in lieu of isomorphism let's talk about the theorem of bijectivity.

We asked her about our approach, the SIT, and then the GIT, she responded, "It seems to be a good idea to me. This theorem could be better understood if one looked at it using the example of the buses, surely." When asked whether something must be taken care of in the group case, she pondered it, and said, "Ah! That it were well defined," and then explained this, both expeditiously and clearly.

\section{DISCUSSION}

As stated in our Approaching the GIT, we expected that students would freely use ERs, partitions, and the ER/P theorem even if they could not formally explain them, and all of them did.

The students who showed an approach to the GIT purely in terms of abstract algebra were not able to develop it and could not explain it. Those who at first 
seemed able to (re)construct the GIT but in the end could not, had trouble when arguing by means of ERs, partitions, and/or ER/P. Conversely, as shown by Carlos's and Carmen's cases, those who could elaborate on ER/P were able to construct the SIT and the GIT and also worked faster, had a clearer view, and seemed more confident. In addition, they were able to construct the GIT from the SIT as we had expected.

Carlos's and Carmen's cases also show that for the GIT it is not necessary to construct the general quotient $G / H$ for $H \unlhd G$, but rather to realize that one must have $\operatorname{Ker}(f) \unlhd G$ in order for $G / \operatorname{Ker}(f)$ to be a group. When students then have to identify the cosets $a \operatorname{Ker}(f), a \in G$, they already have a glimpse of what they are: just the elements of $G$ whose image by $f$ is the same than that of $a$. Thus, we think that a student might benefit from proceeding from $G / \operatorname{Ker}(f)$ to the general quotient group $G / H$ : both normality and the good definition $\bar{f}: G / H \rightarrow G^{2}$ are easier to deal with if $H=\operatorname{Ker}(f)$, and this case is one step further than the abelian case.

Having shown that our approach to the GIT is suitable, we feel it is appropriate to try teaching the (first) group isomorphism theorem the way we proposed here in order to gather further data. Furthermore, Mena-Lorca (2010) has shown that of the dozen or so group homomorphism theorems mentioned at the beginning, all but one have corresponding set theory theorems at their bases, which suggests that a general (categorical) perspective might be used for treating them. In fact, when proving homomorphism theorems for rings, for example, it is natural to look to the corresponding group homomorphism theorem, observe that the group homomorphism is (well) defined, and then just prove its compatibility with the product. Mutatis mutandis, the same is true for group and sets, respectively. This should be further analyzed from a cognitive perspective: going from the general (e.g., sets) to the particular (e.g., groups) might not always the best strategy. For instance, the GIT is an immediate corollary of the fundamental homomorphism theorem for groups (that is, if $\mathrm{N} \unlhd G$, then every group homomorphism $f: G \rightarrow G^{\prime}$ such that $N \subseteq \operatorname{Ker}(f)$, factors uniquely as $f=\bar{f} \circ \pi$, where $\pi: G \rightarrow G / H, \bar{f}: G / H \rightarrow G^{2}$ are defined by $\pi(a)=a N$ and $\bar{f}(a N)=f(a)$, respectively). Nevertheless, in some cases, such as the GIT, we may just be turning to more familiar settings.

\section{NOTES}

1. Research in Undergraduate Mathematics Education Community (1995-2003), founded by Ed Dubinsky.

2. Although they stressed that their teaching preference was to go from the particular to the general, none of them would try first the "fundamental homomorphism for groups": the universal property for $(G / \operatorname{Ker}(f) ; p: G \rightarrow G / \operatorname{Ker}(f), p(a)=a \operatorname{Ker}(f))$.

3. We did not know beforehand that Neubrand (1981) offers a mathematically similar approach, although limited to the GIT.

\section{REFERENCES}

Arnon, I., Cottrill, J., Dubinsky, E., Oktaç, A., Roa, S., Trigueros, M. \& Weller, K. (2014). APOS theory. A framework for research and curriculum development in mathematics education. New York: Springer. DOI 10.1007/978-1-4614-7966-6.

Asiala, M., Brown, A., DeVries, D., Dubinsky, E., Mathews, D., \& Thomas, K. (1996). A framework for research and curriculum development in undergraduate mathematics 
education. In J. Kaput, A. H. Schoenfeld \& E. Dubinsky (Eds.), Research in Collegiate Mathematics Education, II, CBMS Issues in Mathematics Education, 6, 1-32.

Asiala, M., Dubinsky, E., Mathews, D., Morics, S., \& Oktaç, A. (1997). Development of students' understanding of cosets, normality, and quotient groups. Journal of Mathematical Behavior, 16(3), 241-309.

Baker, B., Trigueros, M. \& Hemenway, C. (2001). On transformations of functions. In Proceedings of the Twenty-Third Annual Meeting, North American Chapter of the International Group for PME, Vol. 1 (pp. 91-98).

Bourbaki, N. (1999). Elements of the history of mathematics. Berlin: Springer-Verlag. DOI 10.1007/978-3-642-61693-8.

Brousseau, G. (1997). Theory of didactical situations Mathematics. Dordrecht: Kluwer.

Brown, A., De Vries, D., Dubinsky, E., \& Thomas, K. (1997). Learning binary operations, groups and subgroups. Journal of Mathematical Behavior, 16(3), 187-239.

Burnside, W. (1897). Theory of groups of finite order. Cambridge: Cambridge University Press.

Clark, J., De Vries, D., Hemenway, C., St. John, D., Tolia, G., \& Vakil, R. (1997). An Investigation of students' understanding of abstract algebra (binary operations, groups and subgroups) and the use of abstract structures to build other structures (through cosets, normality and quotient groups). Journal of Mathematical Behavior, 16(3), 181-186.

Dubinsky, E. (1986). Reflective abstraction and computer experiences: A new approach to teaching theoretical mathematics. In G. Lappan \& R. Even (Eds.), Proceedings of the Eighth Annual Meeting of the International Group for the Psychology of Mathematics Education, (pp. 95-121). East Lansing, MI: Michigan State University. Retrieved from http://www.pmena.org/proceedings/PMENA\%2028\%202006\%20Proceedings.pdf

Dubinsky, E. (1991). Reflective abstraction in advanced mathematical thinking. In D. Tall, (Ed.), Advanced mathematical thinking (pp. 95-123). Dordrecht: Kluwer. DOI 10.1007/0-306-47203-1.

Dubinsky, E., Dauterman, J., Leron, U. \& Zazkis, R. (1994). On learning fundamental concepts of group theory. Educational Studies in Mathematics, 27, 267-305.

Dubinsky, E., \& Leron, U. (1994). Learning abstract algebra with ISETL. New York: SpringerVerlag. DOI 10.1007/978-1-4612-2602-4.

Dubinsky, E., \& Lewin P. (1986). Reflective abstraction and mathematics education. Journal of Mathematical Behavior, 5(1), 55-92.

Dubinsky, E., \& McDonald, M. A. (2001). APOS: A constructivist theory of learning in undergraduate mathematics education research. In D. Holton (Ed.), The teaching and learning of mathematics at university level: An ICMI Study (pp. 273-280). Dordrecht: Kluwer Academic Publishers. DOI 10.1007/0-306-47231-7

Dubinsky, E., \& Zazkis, R. (1996). Dihedral groups: A tale of two interpretations. Research in Collegiate Mathematics Education II, CBMS Issues in Mathematics Education 6, 61-82.

Dummit, D., \& Foote, R. (2011). Abstract algebra. Hoboken, New Jersey: John Wiley and Sons.

Fraleigh, J. B. (2003). A first course in abstract algebra. (7th. edition). Upper Saddle River, New Jersey: Pearson.

Galois, E. (1897). Oeuvres mathématiques d'Évariste Galois. Paris: Gauthier-Villars.

Godfrey, D., \& Thomas, M. O. J. (2008). Student perspectives on equation: The transition from school to university. Mathematics Education Research Journal, 20(2), 71-92.

Goetz, J. P., \& Le Compte, M. D. (1988). Etnografía y diseño cualitativo en investigación educativa. Madrid: Morata.

Hamdan, M. (2006). Equivalent Structures on Sets: Equivalence Classes, Partitions and Fiber Structures of Functions. Educational Studies in Mathematics, 62(2), 127-147.

Herstein, I. N. (1999). Abstract Algebra. New York: John Wiley and Sons.

Hazzan, O., \& Leron, U. (1996). Students' use and misuse of mathematical theorems: the case of Lagrange's theorem. For the Learning of Mathematics, 16, 23-26.

Hungerford, T. W. (2003). Algebra. New York: Springer-Verlag.

Iannone, P., \& Nardi, E. (2002). A group as a 'special set'? Implications of ignoring the role of the binary operation in the definition of a group. In A. D. Cockburn \& E. Nardi (Eds.), Proceedings of the 26th Conference of the International Group for the Psychology of Mathematics Education (Vol. 3, pp. 121-128). Norwich, UK.

Ioannou, M. \& Iannone, P. (2011). Students' affective responses to the inability to visualise cosets. Research in Mathematics Education 13(1), 81-82. 
Ioannou, M., \& Nardi, E. (2009). Engagement, abstraction and visualisation: Cognitive and emotional aspects of Year 2 mathematics undergraduates' learning experience in abstract algebra. Proceedings of the British Society for Research into Learning Mathematics, 29(2), 35-40.

Jordan, C. (1869). Mémoire sur les groupes de mouvements, Annali di Math., 11, 167-215 and 332-345.

Jordan, C. (1870). Traité des substitutions et d'équations algébriques. Paris: Gauthier-Villars.

Lajoie, C. (2001). Students' difficulties with the concept of group, subgroup and group isomorphism. In H. Chick, K. Stacey, Jill Vincent \& John Vincent (Eds.): The future of the teaching and learning of algebra, Proceedings of the 12th. ICMI Study Conference. Melbourne: The University of Melbourne.

Lang, S. (2005). Algebra. New York: Addison Wesley.

Larsen, S. (2009). Reinventing the concepts of group and isomorphism: The case of Jessica and Sandra. The Journal of Mathematical Behavior, 28(2-3), 119-137.

Larsen, S. (2013a). A local instructional theory for the guided reinvention of the group and isomorphism concepts. Journal of Mathematical Behavior, 32(4), 712-725.

Larsen, S. (2013b). A local instructional theory for the guided reinvention of the quotient group concept. Journal of Mathematical Behavior, 32(4), 726-742.

Le Compte, M. D., Millroy, W.L., \& Preissle, J. (Eds.) (1992). The handbook of qualitative research in education. San Diego: Academic Press.

Leron, U., \& Dubinsky, E. (1995). An abstract algebra story. American Mathematical Monthly, 102(3), 227-242

Leron, U., Hazzan, O., \& Zazkis, R. (1994). Student's constructions of group isomorphisms. Proceedings of the 18th Annual Conference of the International Group for the Psychology of Mathematics Education, Lisbon, Portugal, vol. 13, pp. 152-159.

Leron, U., Hazzan, O., \& Zazkis, R. (1995). Learning group isomorphism: A crossroads of many concepts. Educational Studies in Mathematics, 29, 153-174.

Mena-Lorca, A. (2010). Estudio epistemológico del teorema del isomorfismo de grupos. (Unpublished doctoral thesis). Centro de Investigación en Ciencia Aplicada y Tecnología Avanzada, Instituto Politécnico Nacional, México. Retrieved from http://www.matedu.cicata.ipn.mx/tesis/doctorado/Mena_2011.pdf

Nardi, E. (1996). The novice mathematician's encounter with mathematical abstraction: tensions in concept-image construction and formalisation. (Doctoral Thesis, University of Oxford). Retrieved from http://ora.ox.ac.uk/objects/uuid:19d55975-7af9-4ed4-ab983be18da31e16

Nardi, E. (2000). Mathematics undergraduate's responses to semantic abbreviations, 'geometric' images and multi-level abstractions in group theory. Educational Studies in Mathematics, 43(2), 168-189.

Neubrand, M. (1981). The homomorphism theorem within a spiral curriculum. International Journal of Mathematical Education, 12(1), 69-74.

Novotná, J., \& Stehlíková, N., \& Hoch, M. (2006). Structure sense for university algebra. In J. Novotná, H. Moraová, M. Krátká, \& N. Stehlíková (Eds.), Proceedings of the 30th Conference of the International Group for the Psychology of Mathematics Education, vol. 4, pp. 249-256. Prague: PME.

Piaget, J., \& García, R. (1982). Psicogénesis e historia de la ciencia. México: Siglo XXI.

Roa-Fuentes, S., \& Oktaç, A. (2010). Construcción de una descomposición genética: Análisis teórico del concepto transformación lineal. Revista Latinoamericana de Investigación en Matemática Educativa, 13(1), 89-112.

Stadler, E. (2011). The same but different - novice university students solve a textbook exercise. In M. Pytlak, T. Rowland, \& E. Swoboda (Eds.), Proceedings of the 7th Conference of European Researchers in Mathematics Education (pp. 2083-2092). Rzeszow, Poland.

Thomas, M.; de Freitas, I.; Huillet, D.; Ju, M.-K.; Nardi, E.; Rasmussen, C.; \& Xie, J. (2015). Key mathematical concepts in the transition from secondary school to university. In Sung Je Cho (Ed.), The Proceedings of the 12th International Congress on Mathematical Education. Intellectual and attitudinal challenges, pp. 265-284. Springer Open.

Trigueros, M., \& Oktaç, A. (2005). La théorie APOS et l'enseignement de l'algèbre linéaire. Annales de Didactique et de Sciences Cognitives, 10, 157-176. Retrieved from 
http://www.irem.univ-paris-diderot.fr/up/annales_de_didactique_et_de_sciences_ cognitives/volume_10/Oktac_Trigueros.pdf

Vergnaud, G. (1981). Quelques orientations théoriques et méthodologiques des recherches françaises en didactique des mathématiques. Recherches en Didactique des Mathématiques, 2, 215-231.

Weber, K (2002). The role of instrumental and relational understanding in proofs about group isomorphisms. Proceedings of the Second International Conference for the Teaching of Mathematics.

Weber, K., \& Larsen, S. (2008). Teaching and learning group theory. In M. Carlson and C. Rasmussen, (Eds.), Making the connection: Research and teaching in undergraduate mathematics education, pp. 137-149. Washington, DC: Mathematical Association of America.

\section{APPENDIX}

\section{Questionnaire}

(" $\leq$ " stands for subgroup, $\overline{\mathrm{z}}$ is the equivalences class of $z$ in $\mathbf{Z}$.)

1. Which of the following are true, if any?

$$
\text { a. } \mathbf{Z}_{5} \leq \mathbf{Z} ; \text { b. } \mathbf{Z}_{5} \leq \mathbf{Z}_{10} ; \text { c. } 6 \mathbf{Z} \leq 3 \mathbf{Z} \text {; d. }\left(\{1,-1\}^{x}\right) \leq(\mathbf{R},+)
$$

2. Let $G=\mathbf{Z}$
a. Assume that $6 \mathrm{Z} \leq G_{y}$ show that $6 \mathrm{Z} \unlhd G$.
b. On $G / 6 \mathrm{Z}$ define $\bar{z}+\overline{z^{\prime}}=\overline{z+z^{4}}$ and explain why it works correctly.

3. Let $G=S_{3}, H=\{(1),(12)\}, K=\{(1)$, (123), (132) $\}$.

a. Show that it is not true that $K \unlhd G$; b. Examine whether $H \unlhd G$; c. Show that $\mathrm{G} / \mathrm{K}$ is not a group.

4. Give five examples of ER for each:

a. Inside mathematics; b. Outside mathematics

5. Explain:

a. What is an ER; b. What is a partition;

c. A result that links ERs and partitions defined over the same set.

6. Define, if possible, a homomorphism.

a. From $\mathbf{Z}_{2}$ to $\mathbf{Z}_{6}$; b. From $\mathbf{Z}_{4}$ to $\mathbf{Z}_{2}$; c. From $\mathbf{Z}_{2}$ to $\mathbf{Z}_{3}$

7. Determine whether $S_{3} \simeq Z_{6}$

8. Let $D_{3}$ be the group of symmetries of a triangle. Determine whether the subgroup of rotations is isomorphic to some $\mathbf{Z}_{n}, n \in \mathbf{N}$.

\section{Script 1}

1. Let $\mathrm{G}$ be a group, $H \leq G$.

A. State the GIT.

B. i. Explain what normal subgroups are needed for.

ii. Describe the quotient group $G / H$.

2. State a theorem that links REs and partitions on a given set. Explain.

3. Let $f: A \rightarrow C$ a function.

i. Show that $f$ defines an ER $R_{f}$ on $A$.

ii. State a theorem that relates the quotient-partition $A / R_{f}$ with another set.

4. A. (If $i$. is achieved)

i. State a relationship between the theorem in 3.ii and the GIT.

ii. Explain what is lacking in 3.ii to be the GIT.

iii. Try to fill in what is lacking. 
B. (If 3.i is not achieved)

i. Let $R_{f}$ be defined on $A$ by $a R_{f} b: f(a)=f(b)$.

a. Show that $R_{f}$ is an ER.

b. What is $A / R_{f}$ ?

5. Assume now that $A$ is a group.

A. Explain how does $R_{f}$ relates to $\operatorname{Ker}(f)$.

B. i. Explain the following: $a R_{\operatorname{Ker}(f)} b ; a^{-1} b \in \operatorname{Ker}(f) ; f\left(a^{-1} b\right)=e^{\prime}$;

$$
f\left(a^{-1}\right) f(b)=e^{v} ; f(a)^{-1} f(b)=e^{t} ; f(b)=f(a)
$$

ii. What conclusion may you extract from i., related to the GIT?

6. Can you distinguish two parts in the GIT?

\section{Script 2}

1. State the GIT. Explain and give an example.

2. What does it mean that a subgroup is normal in a group? What is the purpose of defining normal subgroups?

3. State a theorem that links ERs and partitions defined on a set.

4. Let $f: A \rightarrow B$ be a function.
a. Show that $f$ defines an ER $R_{f}$ on $A$
b. State a theorem that relates the partition $A / R_{f}$ with another set.
c. State a relationship between b. and the GIT. Try to fill in what is lacking.
d. Show that $x R_{f} y: f(x)=f(y)$ defines an ER $R_{f}$ on $A$. What is $R_{f}$ ?
e. Assume now that $f: A \rightarrow B$ is a group homomorphism. How does $R_{f}$ relates to $\operatorname{Ker}(f)$ ?
f. Recall the ER defined on $G$ by $H \unlhd G$. Explain $G / H$. 